\section{Distracción osteogénica maxilar con distractor rígido externo en pacientes con fisura labio-palatina. Revisión bibliográfica}

\section{Maxillary distraction osteogenesis in cleft lip and palate patients using rigid external distractor. A review of the literature}

\begin{abstract}
Resumen
La fisura labio-palatina es la malformación congénita más común entre las anomalías craneofaciales y causa una serie de alteraciones a nivel funcional, fisiológico, estético y social. Una de las características comúnmente asociada a estos pacientes, es la hipoplasia maxilar severa producto de la gran resistencia opuesta por los tejidos cicatrizales de labio y paladar, como consecuencia de la cirugía primaria. Tradicionalmente, el tratamiento ha sido la cirugía ortognática convencional al término del crecimiento, sin embargo, este enfoque ha demostrado tener ciertas limitaciones. La distracción osteogénica mediante un distractor rígido externo ha resultado ser una buena alternativa para el manejo de estos pacientes, ya que permite la distracción gradual del maxilar y de los tejidos asociados, mejorando los resultados a corto y largo plazo. Se realizó una revisión de la literatura actual disponible con el objetivo de describir el alcance del tratamiento de la distracción osteogénica maxilar con distractor rígido externo en los pacientes con fisura labio-palatina e hipoplasia maxilar severa. Se concluye que la distracción osteogénica maxilar con distractor rígido externo es un procedimiento efectivo, confiable y relativamente estable, con mínimas complicaciones asociadas y que podría superar a la cirugía convencional en el manejo de pacientes que requieran un avance maxilar severo.
\end{abstract}

Palabras clave: Osteogénesis por distracción; Labio leporino; Fisura del paladar; Maxilar (fuente: DeCS BIREME).

\begin{abstract}
The cleft lip and palate is the most common congenital malformation among craniofacial anomalies and causes a series of functional, physiological, aesthetic and social alterations. One commonly characteristic associated with these patients is a severe maxillary hypoplasia as a result of the great resistance opposed by scar lip and palate tissue from a primary surgery. Traditionally, the suggested treatment has been conventional orthognathic surgery at the end of skeletal maturity. However, this approach has revealed certain limitations. Distraction osteogenesis using a rigid external distractor has shown to be a good alternative for these patients' treatment, since it allows the gradual distraction of the maxilla and the associated tissues, thereby improving the short and long term results. A
\end{abstract}

\section{Artículo de Revisión}

Noemí Leiva-Villagra ${ }^{1, a}$, Francisca Ayala-Jiménez ${ }^{1, b}$, Carolina Stange-Dempster ${ }^{1, b}$, Vanessa Fuentes-Flores ${ }^{1, b}$

${ }^{1}$ Universidad de Chile, Facultad de Odontología, Unidad de Malformación Cráneofacial, Santiago, Chile.

a Especialista en Ortodoncia y Ortopedia Maxilofacial.

${ }^{\text {b}}$ Cirujana Dentista.

\section{Correspondencia:}

Noemí Leiva

Correo electrónico: leivanoemi@yahoo.com

General Holley 2381, Providencia. Santiago, Chile.

\section{Coautores:}

Francisca Ayala

francisca@ayalajimenez.com

Carolina Stange

carostange@hotmail.com

Vanessa Fuentes

vane.fuentes.f@gmail.com

Editor:

Yuri Castro Rodríguez

Universidad Nacional Mayor de San Marcos, Perú.

Conflicto de intereses: los autores declaran no tener conflictos de interés.

Fuente de financiamiento: autofinanciado.

Recibido: 22/03/2019

Aceptado: 09/05/2019

Publicado: 12/09/2019 
review of the available literature was performed to describe the maxillary distraction osteogenesis treatment scope using a rigid external distractor in cleft lip and palate patients with severe maxillary hypoplasia. It was concluded that maxillary distraction osteogenesis using a rigid external distractor is an effective, reliable and relatively stable procedure, with minimal associated complications. This could be a better alternative compared to conventional surgery for patients' care having severe maxillary hypoplasia.

Keywords: Distraction osteogenesis; Cleft lip; Cleft palate; Maxilla (source: $\mathrm{MeSH}$ NLM).

\section{Introducción}

La fisura labio-palatina (FLP) es la malformación congénita más común entre las anomalías craneofaciales ${ }^{1}$, presentando una incidencia a nivel mundial de 1 cada 700 nacidos vivos. Es causada por la fusión inadecua$\mathrm{da}$ o inexistente de los procesos embrionarios que dan origen a la formación de las cavidades nasales y cavidad oral, alterando así, la anatomía normal del labio superior y/o paladar. La FLP causa una serie de alteraciones a nivel funcional, fisiológico, estético y social ${ }^{2}$, que impactan directamente en la calidad de vida del paciente.

En cuanto a la etiología, existe consenso respecto a su naturaleza multifactorial, existiendo predisposición genética e influencia ambiental. Dentro de los factores de riesgo descritos en la literatura, podemos encontrar el consumo de cigarro y/o alcohol, el uso de drogas anti epilépticas o corticoesteroides administrados de forma tópica o sistémica, inadecuada nutrición de la madre durante el embarazo y bajo nivel socioeconómico ${ }^{3}$.

Los pacientes con FLP frecuentemente presentan una falta del desarrollo del tercio medio facial y una hipoplasia maxilar severa atribuida tanto a la deficiencia congénita del crecimiento de las estructuras en relación a la fisura, como a los efectos negativos de las cirugías realizadas en la infancia ${ }^{4}$, lo que conlleva características típicas como retrusión severa del tercio medio facial, perfil cóncavo, base nasal ancha, punta nasal invertida y ángulo nasolabial disminuido.

A nivel intraoral es común encontrarse con diversos grados de maloclusiones esqueletales tipo III, mordida invertida severa, mordida cruzada unilateral o bilateral, alteraciones del arco dentario maxilar en sentido sagital, vertical y transversal ${ }^{5}$, curva de Spee aumentada, múltiples agenesias dentarias (especialmente en la zona de la fisura o adyacente a esta), comunicación oronasal y fisura residual. Además, como resultado de la hipoplasia maxilar severa, la mandíbula tiende a rotar hacia arriba y adelante con la subsecuente disminución en la dimensión vertical, pérdida de altura facial anterior, pseudoprognatismo e inclinación del plano oclusal ${ }^{6}$. Otras manifestaciones se dan a nivel de la fonación, donde existen alteraciones producto de la incompetencia velofaríngea y comunicación oronasal ${ }^{7}$.

Dentro de las alternativas de tratamiento para estos pacientes, se ha aceptado el uso de la máscara de tracción a edades tempranas, sin embargo, esta no permite un avance maxilar mayor a 4 o $5 \mathrm{~mm}$. Se ha reportado en la literatura, que un 25 a $50 \%$ de los pacientes con hipoplasia maxilar severa como consecuencia de FLP, requieren de un tratamiento quirúrgico para lograr un resultado estético y funcional $6,8,9$.

El tratamiento tradicional para esta anomalía ha sido desde 1977 la combinación entre ortodoncia/ortopedia dentomaxilar y cirugía ortognática con osteotomía Le Fort $1^{10}$, sin embargo, este enfoque presenta ciertas limitaciones. Es una técnica que requiere haber completado la madurez esqueletal; sin embargo, muchas veces la desarmonía facial es tan severa que, esperar hasta el término del crecimiento puede resultar insoportable para el paciente, principalmente debido a las posibles repercusiones en edades cruciales para el desarrollo psicosocial del individuo ${ }^{5,11}$.

Diversos autores concuerdan en que cuando el avance maxilar requerido es mayor a $10 \mathrm{~mm}$, la cirugía convencional no sería el procedimiento más indicado ${ }^{12}$. La tasa de recidiva asociada a un avance maxilar inmediato es del 20 al 25\%, principalmente dada por la resistencia de los tejidos cicatrizales palatinos y del labio superior, que al no tener fibras de elastina y poseer fibras colágenas muy ordenadas, se comportan como un tejido rígido y tenso que opone gran resistencia al avance maxilar inmediato y mantenido en el tiempo ${ }^{13-15}$. Por último, la cirugía convencional puede gatillar o empeorar la insuficiencia velofaríngea (IVF), que es uno de los principales problemas del habla asociado a los pacientes con FLP ${ }^{16}$.

La distracción osteogénica (DO) se ha presentado como una buena alternativa en el tratamiento de estos pacientes, ya que ofrece resultados funcionales y estéticos más predecibles y según algunos autores, con menor recidiva y mayor estabilidad en el tiempo ${ }^{4,7}$. Esta técnica fue descrita por primera vez en los años 50 por Ilizarov 17 en Rusia. Él describió el principio de tensión-estrés, según el cual, al aplicar una tensión gradual y estable sobre un tejido vivo, se genera un estrés que puede estimular y mantener la regeneración y crecimiento activo de ciertas estructuras.

En vista de que la cirugía ortognática convencional en muchos casos no es el tratamiento más adecuado para estos pacientes, la DO se ha presentado como una atractiva y confiable alternativa, tanto para pacientes en crecimiento que presentan alteraciones estéticas y funcionales severas, como para pacientes adultos.

El objetivo de esta revisión bibliográfica es describir según la literatura científica disponible el alcance del tratamiento de la hipoplasia maxilar severa como consecuencia de la FLP mediante DO y distractor rígido externo. 


\section{Desarrollo}

La primera aplicación de la DO en huesos craneofaciales fue realizada por McCarthy et al. ${ }^{18,}$ donde describieron la elongación de la mandíbula en pacientes con deformidades mandibulares severas. Posteriormente, Cohen et al. ${ }^{19}$ fueron los primeros en reportar la aplicación exitosa de esta ténica en pacientes con FLP e hipoplasia severa del tercio medio facial. Finalmente, Polley y Figueroa ${ }^{20}$ introdujeron la técnica de avance maxilar con DO utilizando un distractor rígido externo o RED (por sus siglas en inglés "rigid external distractor"), que desde entonces ha sido ampliamente utilizada.

En su publicación en el año 1997, Polley y Figueroa ${ }^{20}$ establecen las indicaciones para la DO maxilar con RED, entre las cuales se encuentran:

- Deficiencia maxilar severa en sentido transversal, vertical y horizontal

- Requerir un avance maxilar mayor a 8-10 mm

- Fisuras palatinas con tejido cicatrizal severo y colgajo faríngeo

- Fisura labio-palatina bilateral con premaxila inexistente, rudimentaria o desdentada

- Edad mínima que asegure una dentición temporal completa

- Morfología y posición mandibular normales

- Relaciones mandíbulo-cervicales normales

- Cráneo intacto

El RED se compone de una estructura rígida externa y una férula rígida intraoral que es cementada previa o durante la cirugía a los segundos molares temporales, primeros/segundos premolares o primeros molares permanentes. En la figura 1, se muestran las fotografías preoperatorias, perioperatorias y 6 años postoperatorias de un paciente con FLP tratado con DO y RED, mediante anclaje dentario.
En un principio, Polley y Figueroa ${ }^{20}$ establecieron que el sistema RED dependía de un anclaje dentario para transmitir las fuerzas de distracción al maxilar, y que los cambios no deseados en el componente dental eran mínimos. No obstante lo anterior, con el tiempo se fueron reportando compensaciones dentoalveolares importantes, por lo que se comenzó a incorporar elementos de anclaje esqueletal para reducir los efectos a nivel dentario.

Posteriormente, Polley y Figueroa ${ }^{21}$ modificaron el diseño del dispositivo intraoral, incorporándole soporte esqueletal mediante tornillos de anclaje óseo, que eran conectados mediante alambre a la porción anterior del dispositivo intraoral. Esta modificación disminuyó la aplicación directa de la fuerza a la dentición. Actualmente, la técnica más utilizada es el uso de placas fijadas mediante tornillos a nivel paranasal, evitando el daño a los gérmenes dentarios y otorgando anclaje esqueletal al distractor. En la figura 2 se expone un RED utilizando este tipo de anclaje ${ }^{22}$.

\section{Técnica quirúrgica}

La mayoría de los estudios se basó en el protocolo descrito por Polley y Figueroa ${ }^{20}$. Este consiste en una osteotomía Le Fort I alta con disyunción septal y ptérigomaxilar, sin "down fracture", evitando los gérmenes dentarios. Algunos autores realizaron una osteotomía Le Fort I convencional generalmente al existir dentición permanente ${ }^{23-28}$. El maxilar es completamente movilizado sin ser desplazado ni reposicionado como sucede en la cirugía ortognática convencional. Se inserta la placa de tracción paranasal y posterior al cierre de los tejidos blandos, se realiza la instalación de la porción externa del dispositivo. Esta, es fijada al cráneo mediante tres a cuatro tornillos bilaterales, posicionados 3 a $4 \mathrm{~cm}$ por encima del borde del hélix, con el plano horizontal del dispositivo paralelo al plano de Frankfurt. Luego, los ganchos de tracción externos del dispositivo intraoral o los elementos de anclaje esqueletal son conectados mediante alambres de tracción a la barra horizontal del
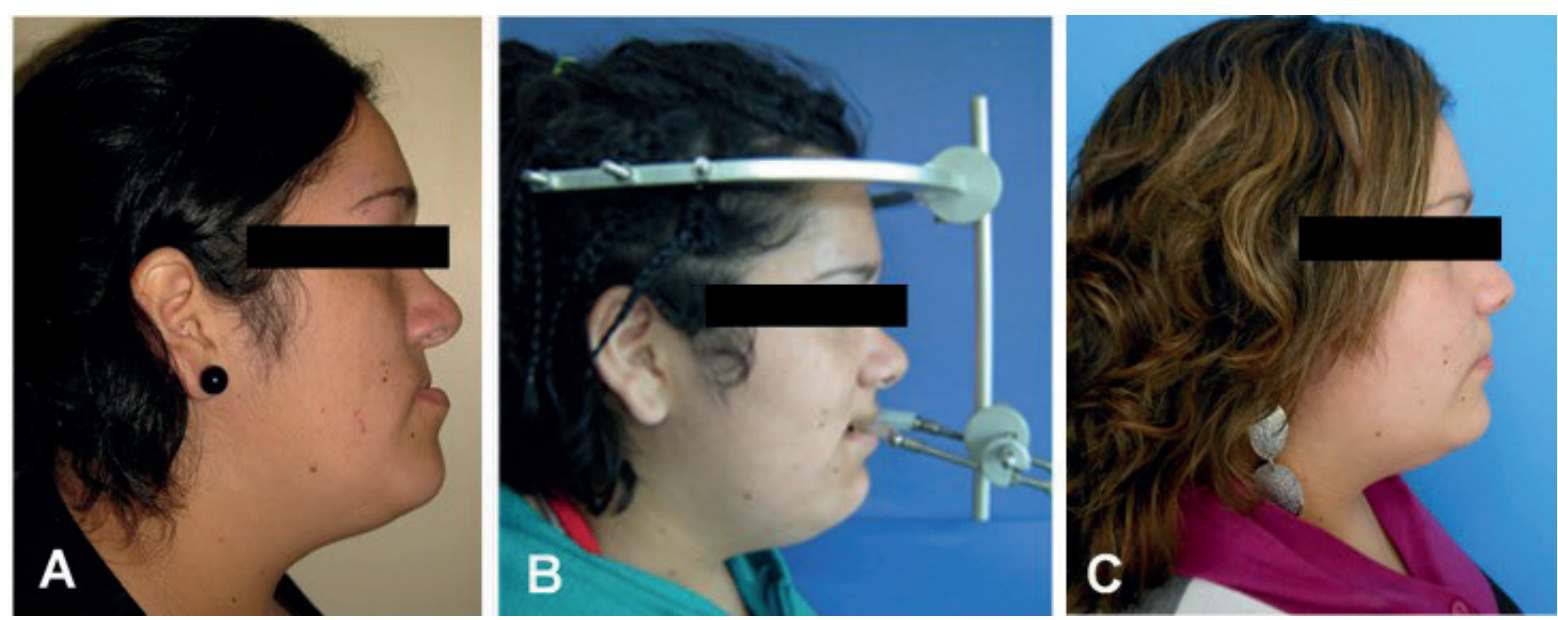

Figura 1. Distracción osteogénica con distractor rígido externo y anclaje dentario en paciente con fisura labio-palatina. A. Preoperatorio. B. Período de consolidación. C. A los 6 años postoperatorio 


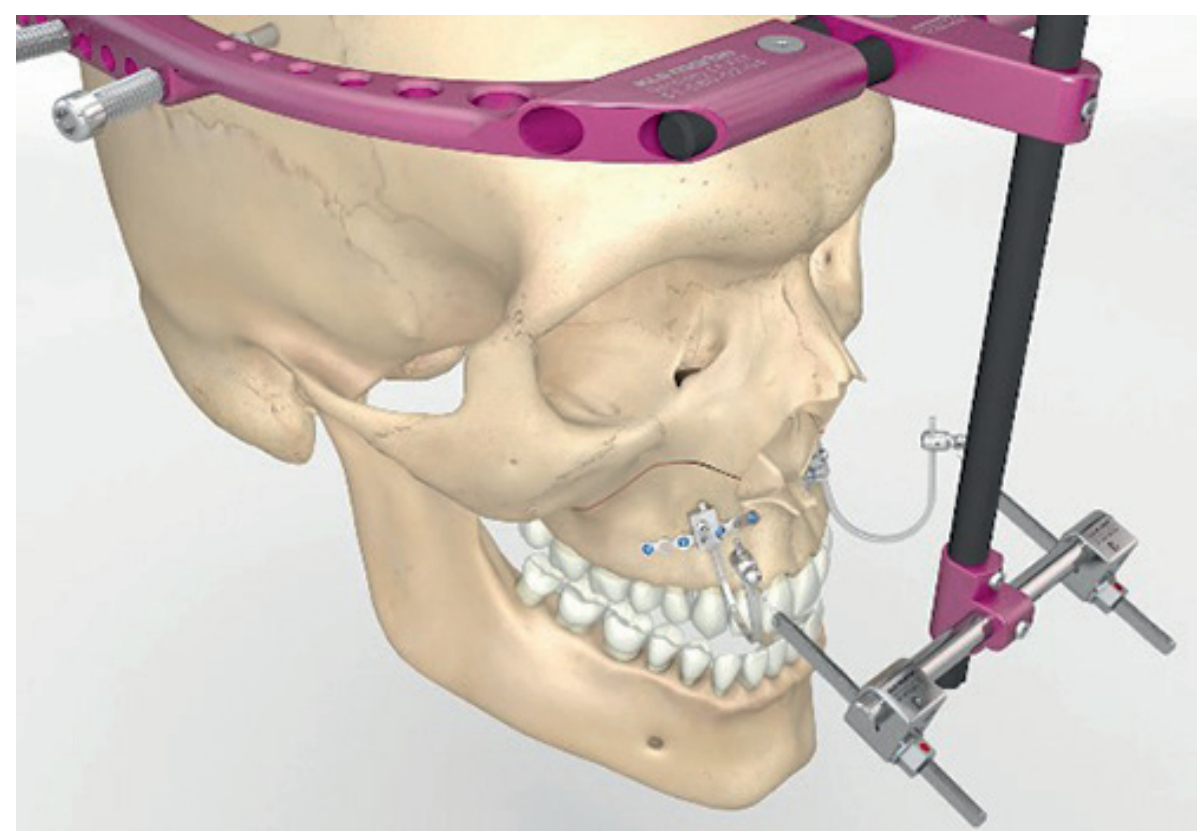

Figura 2. Distractor rígido externo con anclaje óseo parasanal. Fuente: KLS Martin Group ${ }^{21}$

distractor, desde donde se puede manejar el vector de distracción ${ }^{20}$.

Es importante tomar en cuenta el centro de masa del maxilar para lograr el vector de distracción planificado; éste se considera localizado en los ápices de los premolares maxilares. Si se requiere un avance lineal, paralelo al plano oclusal funcional, la línea de acción de la fuerza de distracción debería pasar a través del centro de masa (en una vista sagital) y ser paralela al plano oclusal funcional. Si se requiere una rotación del maxilar en sentido horario, la línea de acción de la fuerza aplicada debería pasar por arriba del centro de masa, y ser paralela al plano oclusal funcional. Asimismo, si se requiere un movimiento maxilar anti horario, la línea de acción de la fuerza aplicada debe pasar por debajo del centro de masa, también paralela al plano oclusal funcional ${ }^{29}$.

Después de realizar la instalación quirúrgica del dispositivo, existe un período de latencia de entre 2 a 7 días, durante el cual no existe activación. Posterior a éste, se inicia la fase activa de distracción, a una tasa de 1 a 2 $\mathrm{mm} /$ día, hasta lograr la cantidad de movimiento horizontal y vertical deseado. Le sigue el período de consolidación, en el cual, el dispositivo se mantiene en posición de forma pasiva por un tiempo que puede variar entre 4 a 16 semanas ${ }^{23-28,30-34}$. Finalmente, el distractor es retirado y se indica el uso de máscara facial en un rango de 6 a 36 semanas, con el objetivo de lograr una mejor estabilidad en el tiempo y disminuir el riesgo de recidiva 23,25,27,30-33. Solo un estudio utilizó elásticos de tracción clase III en conjunto con ortodoncia postoperatoria como reemplazo de la máscara facial ${ }^{26}$.

Actualmente, el advenimiento de la tecnología CAD/ CAM ("Computer-Aided Design/Computer-Aided Manufacturing"), ha significado importantes avances tanto en el diagnóstico como en la planificación del tratamiento. La introducción de la tomografía computari- zada (TC) en combinación con sofisticados softwares de procesamiento de imágenes, han permitido a los profesionales obtener la anatomía del paciente y poder planificar los procedimientos con gran precisión. La reconstrucción digital de cortes bidimensionales en un modelo tridimensional (3D), ha sido particularmente útil en la evaluación de anomalías craneofaciales, ya que permite relacionar la posición de distintas estructuras anatómicas distantes entre sí, y así, poder evaluar la anomalía en los tres planos del espacio ${ }^{35}$.

Asimismo, esta información puede ser utilizada para el confeccionamiento de un modelo 3D en acrílico, a través de la estereolitografía, lo que permitiría, por ejemplo, la adaptación de las placas paranasales previo a la cirugía, disminuyendo el tiempo intraoperatorio; no obstante, esta técnica requiere de mayor cantidad de tiempo y es costosa para el paciente ${ }^{36}$.

Otra aplicación es la utilización de un software para la planificación virtual del tratamiento a través de las imágenes 3D obtenidas mediante la TC, esto permitiría al tratante, visualizar los resultados esperados y los parámetros de movimiento de la corrección propuesta, determinando con gran precisión el vector de distracción requerido. Todo lo anterior se traduce en un tratamiento con resultados más predecibles ${ }^{35}$.

\section{Avance esqueletal}

En relación a los cambios lineales logrados con la DO y RED, el avance maxilar en el plano sagital fue significativo, en un promedio de 13,8 $\mathrm{mm}$, sin embargo, la mayoría de los autores utilizó puntos cefalométricos y planos de referencia diferentes, por lo que la comparación entre cada uno de ellos se hace difícil ${ }^{23-28,30-33}$. En la tabla se presenta un resumen del avance maxilar sagital logrado por los principales estudios, junto con los puntos y planos cefalométricos que utilizaron. 
Tabla. Avance maxilar obtenido según los puntos y planos cefalométricos utilizados en los principales estudios

\begin{tabular}{|c|c|c|c|c|c|c|c|c|c|c|}
\hline & $\begin{array}{l}\text { Gürsoy et } \\
\text { al. } 2010^{30}\end{array}$ & $\begin{array}{l}\text { Sahoo et } \\
\text { al. } 2014{ }^{31}\end{array}$ & $\begin{array}{l}\text { Dua et al. } \\
2014{ }^{33}\end{array}$ & $\begin{array}{l}\text { Meazzini } \\
\text { et al. } 2015^{24}\end{array}$ & $\begin{array}{l}\text { Kim et al. } \\
2015^{25}\end{array}$ & $\begin{array}{l}\text { Sahoo et } \\
\text { al. } 2015^{32}\end{array}$ & $\begin{array}{l}\text { Taha et } \\
\text { al. } 2016^{34}\end{array}$ & $\begin{array}{l}\text { Jena et } \\
\text { al. } 2016^{26}\end{array}$ & $\begin{array}{l}\text { Sawada et } \\
\text { al. } 2017^{27}\end{array}$ & $\begin{array}{l}\text { Painatt et } \\
\text { al. } 2017^{28}\end{array}$ \\
\hline $\begin{array}{l}\text { Número de } \\
\text { pacientes }\end{array}$ & 13 & 15 & 9 & 22 & 21 & 10 & 30 & 12 & 6 & 11 \\
\hline $\begin{array}{l}\text { Edad promedio } \\
\text { (años) }\end{array}$ & $\begin{array}{l}\text { Varones: } \\
\text { 13,6 } \pm 1,3 \\
\text { Mujer.: } 12,8 \\
\pm 1,2\end{array}$ & $\begin{array}{l}13,07 \pm \\
2,28\end{array}$ & $\begin{array}{l}18,8 \\
\text { (rango } \\
17-22)\end{array}$ & $\begin{array}{l}\text { Crecim.: } \\
\text { 10,4 } \\
\text { Adulto: } \\
24,4\end{array}$ & $\begin{array}{l}10 \\
\text { (rango } \\
8-14 \text { ) }\end{array}$ & $\begin{array}{l}\text { 15,1 } \\
\text { (rango } \\
11,3-19,8 \text { ) }\end{array}$ & $\begin{array}{l}17,13 \pm \\
4,9\end{array}$ & $\begin{array}{l}18,91 \\
\pm 1,04\end{array}$ & $\begin{array}{l}17,7 \text { (rango } \\
12-23 \text { ) }\end{array}$ & $\begin{array}{l}19,2 \\
\text { (rango } \\
15-30 \text { ) }\end{array}$ \\
\hline $\begin{array}{l}\text { Avance maxilar } \\
\text { sagital prome- } \\
\text { dio y puntos } \\
\text { cefalométricos } \\
\text { utilizados }\end{array}$ & $\begin{array}{l}12 \mathrm{~mm} \\
\text { (dist. punto } \\
\text { A, ANS y } \\
\text { PNS a la } \\
\text { vertical de } \\
\text { referencia) }\end{array}$ & $\begin{array}{l}10 \mathrm{~mm} \\
\mathrm{Co}-\mathrm{A}\end{array}$ & $\begin{array}{l}13,4 \mathrm{~mm} \\
\text { A-X }\end{array}$ & $\begin{array}{l}\text { Crecim.: } \\
\text { 22,2 mm } \\
\text { Adulto: } \\
\text { 17,7 mm } \\
\text { (desplaz. } \\
\text { punto A) }\end{array}$ & $\begin{array}{l}\text { No me- } \\
\text { dido }\end{array}$ & $\begin{array}{l}9,3 \mathrm{~mm} \\
\text { Vert N-A }\end{array}$ & $\begin{array}{l}8,5 \mathrm{~mm} \\
\text { (dist. } \\
\text { punto A } \\
\text { al eje y) } \\
11 \mathrm{~mm} \\
\text { (dist. pun- } \\
\text { to ANS al } \\
\text { eje y) }\end{array}$ & $\begin{array}{l}10 \mathrm{~mm} \\
\text { (dist. li- } \\
\text { neal entre } \\
\text { el punto } \\
\text { Ptm y } \\
\text { punto M }\end{array}$ & $\begin{array}{l}8,5 \mathrm{~mm} \\
\text { (desplaz. } \\
\text { punto } \\
\text { ANS a lo } \\
\text { largo del } \\
\text { plano de } \\
\text { Frankfurt }\end{array}$ & $\begin{array}{l}14,6 \mathrm{~mm} \\
\text { Co-A }\end{array}$ \\
\hline $\begin{array}{l}\text { Avance maxilar } \\
\text { angular } \\
\text { promedio }\end{array}$ & $\begin{array}{ll}\text { SNA: } & 13^{\circ} \\
\text { ANB: } & 13^{o}\end{array}$ & $\begin{array}{l}\text { SNA: } 8^{\circ} \\
\text { ANB: } 3,7^{\circ}\end{array}$ & $\begin{array}{l}\text { SNA: } \\
10,7^{\circ} \\
\text { ANB: } \\
11,2^{\circ}\end{array}$ & $\begin{array}{l}\text { Crecim.: } \\
\text { SNA: } 16,5^{\circ} \\
\text { Adulto: } \\
\text { SNA: } 13,9^{\circ}\end{array}$ & $\begin{array}{l}\text { SNA: } \\
13,1^{\circ} \\
\text { ANB: } 13,3\end{array}$ & $\begin{array}{l}\text { SNA: } 10,7^{\circ} \\
\text { ANB: } 7,7^{\circ}\end{array}$ & SNA: $9,6^{\circ}$ & $\begin{array}{l}\text { SNM: } \\
10,1^{\circ}\end{array}$ & no medido & SNA: $13,9^{\circ}$ \\
\hline
\end{tabular}

Puntos cefalométricos y parámetros lineales y angulares. A: punto más profundo de la concavidad anterior del maxilar; B: punto más profundo de la concavidad anterior de la mandíbula; S: punto silla turca; N: punto nasion; Ptm: punto pterigomaxilar; M: centro de la pre-maxila; ANS: espina nasal anterior (por sus siglas en inglés "anterior nasal spine"); PNS: espina nasal posterior (por sus siglas en inglés "posterior nasal spine"); Co-A: distancia lineal entre el punto Condilion (Co) y el punto " $\mathrm{A}$ " proyectados perpendicularmente sobre el plano de Frankfurt, representa la longitud maxilar efectiva; A-X: distancia entre el punto " $\mathrm{A}$ " y la vertical de referencia a lo largo de la línea horizontal de referencia; Vert N-A: distancia lineal entre la vertical

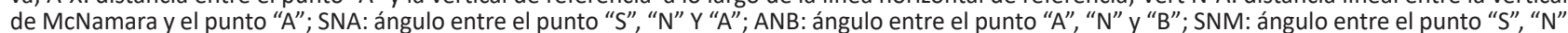
y " $\mathrm{M}$ ". Líneas de referencia. Vertical de referencia: línea que atraviesa el punto " $S$ " y es perpendicular a la línea horizontal de referencia; Horizontal de referencia: línea que atraviesa el punto "N" y presenta una rotación superior de 7o con respecto a la línea S-N; Vertical de McNamara: línea perpendicular al plano de Frankfurt que pasa por el punto "N"; Eje y: línea perpendicular a la línea S-N, que pasa por el punto "S"

Algunos de los parámetros utilizados para determinar dicho avance fueron: el desplazamiento del punto A 23,24,30,32,33, la longitud maxilar medida entre los puntos Condilion y A (Co-A) ${ }^{28,31}$, el desplazamiento del punto ANS (espina nasal anterior por sus siglas en inglés) 27,30

El desplazamiento vertical del maxilar solo se describe en seis estudios ${ }^{24,27,30-33}$, en un rango de 2 a $6,8 \mathrm{~mm}$ hacia abajo. El resto de las publicaciones no hace referencias al respecto $23,25,26,28,34$.

En relación a los cambios angulares, hubo un aumento significativo en el ángulo SNA (rango de 8 a 16,50) durante el postoperatorio inmediato, así como también en el ángulo ANB (rango de 3,7 a 13,3), lo que indica que hubo un avance maxilar efectivo y una mejoría en la relación maxilo-mandibular ${ }^{23-25,28,30-34}$. En cuanto al ángulo SNB, éste no sufrió cambios significativos en la mayoría de los estudios. Doucet et al. ${ }^{23}$, evaluaron específicamente el efecto de la $\mathrm{DO}$ maxilar en la posición vertical y sagital de la mandíbula, y encontraron que cuando el avance maxilar era igual o superior a los 15 $\mathrm{mm}$, la mandíbula tendía a rotar en sentido horario, sin embargo, esta rotación no fue significativa.

\section{Recidiva esqueletal}

En general, los valores postoperatorios logrados tienden a recidivar sobre todo cuando se evalúan a largo plazo, sin embargo, en la mayoría de las publicaciones se logró un resultado estético y una oclusión estable y funcional en el tiempo ${ }^{23,24,26-28,30-34}$. Gürsoy et al. ${ }^{30}$ analizaron 13 pacientes prepuberales con FLP, tratados con DO y RED, y encontraron que los cambios dentoesqueletales logrados fueron estables durante el primer y segundo año de seguimiento, pero fueron cambiando hacia los valores preoperatorios en el largo plazo (2-5 años). Esto se explica por una recidiva en el componente dental y por el crecimiento diferencial entre el maxilar y la mandíbula. Cabe rescatar que si bien hubo recidiva en los valores, la estética facial se mantuvo casi por completo y el ángulo ANB y overjet se mantuvieron en valores positivos.

Otro factor a considerar es la edad del paciente al momento de la distracción. Meazzini et al. ${ }^{24}$ analizaron la estabilidad a corto y largo plazo en pacientes adultos y en crecimiento. Al primer año de seguimiento encontraron que los resultados eran muy estables en el grupo adulto, no obstante, en el grupo en crecimiento se observó una recidiva horizontal del $16 \%$ y posterior a los 5 años de seguimiento, el valor ascendió a un 26\%. Lo anterior está en concordancia con lo reportado por Kim et al. ${ }^{25}$ quienes encontraron que el único factor significativo entre dos grupos de pacientes sometidos al mismo protocolo quirúrugico de DO maxilar con RED, y con resultados diferentes (recidiva y no recidiva) fue la edad. Es importante considerar que, si bien los porcentajes de recidiva tienden a ser mayores en los pacientes prepuberales, la DO avanza al maxilar a una posición más anterior que si el paciente no hubiese sido operado. Esto se explica porque en los pacientes con FLP, el potencial de crecimiento maxilar se encuentra disminuido y el crecimiento que pudiese lograr de forma espontánea es mucho menor. Además, algunos autores sostienen que el crecimiento del maxilar puede verse afectado por 
la distracción propiamente tal, ya que la sutura pterigomaxilar es un importante centro de crecimiento y se encuentra en la línea de la osteotomía y en la zona de neoformación ósea, lo que puede impactar negativamente en su desarrollo posterior ${ }^{25}$.

Algunos autores atribuyen la tendencia a la recidiva a la resistencia que oponen los tejidos cicatrizales palatinos y del labio superior en los pacientes con FLP y a la magnitud del desplazamiento maxilar, ya que se ha visto en algunos estudios que existe una correlación positiva entre la cantidad de avance maxilar y la magnitud de la recidiva ${ }^{28,31}$.

En vista de que la tendencia a la recidiva es clara, múltiples autores recomiendan realizar la DO maxilar con un porcentaje de sobrecorrección anterior en un rango de un $15 \%$ a $40 \%$, sobre todo en pacientes en crecimiento $25,28,31,32$. Solamente un estudio ${ }^{30}$ no recomienda realizar sobrecorreción ya que propone que el llevar al maxilar a la posición adulta durante la adolescencia pudiese deteriorar las funciones masticatorias. El resto de los autores $23,24,26,27,33$ no hacen referencia al respecto.

Otro factor que ha sido mencionado en la literatura, es la estabilidad oclusal lograda posterior a la DO. Es de gran importancia que la oclusión final presente un adecuado entrecruzamiento anterior (overjet y overbite), ya que este, representa la llave para la estabilidad de los resultados en el tiempo ${ }^{37}$.

\section{Cambios en tejido blando}

En general, existe consenso respecto a la mejoría significativa que produce la DO maxilar en el complejo nasomaxilar siendo mayor que la que produce la cirugía ortognática convencional. Algunos autores reportan que la osteotomía Le Fort I alta además de prevenir el daño a los ápices dentarios, permite la movilización de segmentos óseos más extensos logrando mejorar el soporte parasanal que se encuentra disminuido en este tipo de pacientes ${ }^{28,31}$.

El perfil blando experimentó cambios importantes pasando de ser cóncavo a recto o convexo, mejorando la apariencia estética considerablemente. El labio superior adquirió una posición más anterior, contribuyendo a disminuir su grosor y a la conformación de un perfil armónico.

Los parámetros más comúnmente evaluados fueron el ángulo nasolabial, y el ángulo de convexidad facial. Hubo un aumento significativo de estos parámetros en un promedio de $17,83^{\circ}{ }^{28,31-33}$ y $17,21^{\circ}{ }^{31-33}$ respectivamente, manteniéndose relativamente estables al primer año de seguimiento.

\section{Función velofaríngea y nasorespiratoria}

Es sabido que la cirugía de avance maxilar convencional podría gatillar o empeorar una IVF preexistente, condición altamente prevalente en los pacientes con FLP y que corresponde al principal problema asociado al habla 16,34. Esto se podría explicar por el desplazamiento anterior del paladar blando (con menor movilidad debido al tejido cicatrizal) que genera un gran espacio entre el paladar y la pared faríngea empeorando la función velofaríngea, especialmente la hipernasalidad.

Por otro lado, se ha reportado que un avance maxilar gradual, como el que se produce con la DO, podría contribuir a la adaptación de los tejidos blandos a los cambios esqueletales y ayudar a prevenir el empeoramiento de la IVF.

En esta revisión de la literatura, solo dos estudios ${ }^{28,34}$ evaluaron cambios en la nasofaringe posterior a la DO maxilar. Al respecto, ambos reportaron un aumento significativo en la profundidad de la nasofaringe debido al desplazamiento anterior de la espina nasal posterior y paladar blando producto de la DO maxilar. Este hecho favorece la respiración nasal y presenta una correlación positiva con la cantidad de avance maxilar realizado. En ambos estudios, los resultados fueron relativamente estables al primer año de seguimiento.

En cuanto a la función velofaríngea, Taha et al. ${ }^{34}$ reportaron que un $87 \%$ de los pacientes evaluados presentaron algún grado de IVF previa a la DO. En adición, un $46,6 \%$ experimentó un deterioro en la hipernasalidad posterior al procedimiento, principalmente en pacientes con algún grado de hipernasalidad previa a la cirugía. De los pacientes que presentaron hiponasalidad preexistente, un $67 \%$ obtuvo mejorías posteriores a la DO maxilar.

\section{Complicaciones}

Las complicaciones asociadas a la DO maxilar fueron escasas y menores. La principal tuvo que ver con el aflojamiento del dispositivo por accidente (solamente en tres pacientes), por lo cual tuvo que ser reposicionado 23,27. Sahoo et al. ${ }^{32}$ reportaron pérdida de cabello en las zonas de los tornillos extraorales en el $100 \%$ de su muestra. Kim et al. ${ }^{25}$ no reportaron ninguna complicación asociada al procedimiento. El resto de los autores no hicieron referencia a la presencia u ausencia de complicaciones ${ }^{24,26,28,30,31,33,34}$.

\section{Conclusiones}

El tratamiento de los pacientes con hipoplasia maxilar severa como consecuencia de FLP es complejo y habitualmente el enfoque tradicional de la cirugía ortognática no cumple con todas las expectativas del tratante y del paciente. La literatura actual muestra que la DO maxilar con RED es un procedimiento versátil, confiable, efectivo y relativamente estable a corto y largo plazo, con mínimas complicaciones asociadas, siendo altamente recomendable para el manejo de los pacientes con FLP e hipoplasia maxilar severa.

\section{Referencias bibliográficas}

1. Vinay C, Sudhakara R, Uloopi KS, Madhuri V, Chandra R. Craniofacial features in Goldenhar syndrome. J Indian Soc Pedod Prev Dent. 2009;27(2):121-124. DOI: 10.4103/0970-4388.55339.

2. Cordero E, Correa S, Pantoja R. Prevalencia de pacientes con fisuras labio palatinas tratadas en el hospital San 
Borja Arriarán en Santiago de Chile, en el marco del plan auge. Int J Odontostomat. 2015;9(3):469-473.

3. Kloukos D, Fudalej P, Sequeira-Byron P, Katsaros C. Maxillary distraction osteogenesis versus orthognathic surgery for cleft lip and palate patients. Cochrane Database Syst Rev. 2018;10(8):CD010403. DOI: 10.1002/14651858.CD010403.pub3.

4. Richardson S, Krishna S, Bansal A. Use of repeat anterior maxillary distraction to correct residual midface hypoplasia in cleft patients. J Korean Assoc Oral Maxillofac Surg. 2017;43(6):407-414. DOI: 10.5125/ jkaoms.2017.43.6.407.

5. Wada T, Miyazaki T. Treatment principles for the changing arch form in children with complete unilateral cleft lips and palates. Cleft Palate J. 1976;13:273-283.

6. Scolozzi P. Distraction osteogenesis in the management of severe maxillary hypoplasia in cleft lip and palate patients. J Craniof Surg. 2008;19(5):1199-1214. DOI: $10.1097 /$ SCS.0b013e318184365d.

7. Sahoo NK, Jayan B, Roy ID, Desai A. Evaluation of the hard and soft tissue changes after maxillary advancement with rigid external distractor in unilateral cleft lip and palate induced maxillary hypoplasia. Med J Armed Forces India. 2014;71(2):355-361. DOI: 10.1016/j.mjafi.2013.12.016.

8. Ross RB. Treatment variables effecting facial growth in complete unilateral cleft lip and palate. Cleft Palate J. 1987;24(1):5-77.

9. Linton JL. Comparative study of diagnostic measures in borderline surgical cases of unilateral cleft lip and palate and noncleft class III malocclusions. Am J Orthod Dentofacial Orthop. 1998;113(5):526-537.

10. Bell WH, McBride KL. Correction of the long face syndrome by Le Fort I osteotomy. A report on some new technical modifications and treatment results. Oral Surg Oral Med Oral Pathol. 1997;44(4):493-520.

11. Park YW, Kwon KJ, Kim MK. Long-term follow-up of early cleft maxillary distraction. Maxillofac Plast Reconstr Surg. 2016;38(1):20. DOI: 10.1186/s40902-0160069-x.

12. Cheung LK, Chua HD, Hägg MB. Cleft maxillary distraction versus orthognatic surgery: clinical morbidities and surgical relapse. Plast Reconstr Surg. 2006;118(4):9961008. DOI: 10.1097/01.prs.0000232358.31321.ea.

13. Bevilacqua RG, Ritoli EL, Kang C, Mabry K, Castiglione CL. Midmaxillary internal distraction osteogenesis: ideal surgery for the mature cleft patient. Plast Reconstr Surg. 2008;121(5):1768-1778. DOI: 10.1097/PRS. 0b013e31816a9f2d.

14. Hoff JW, Maltha JC, Kuijpers-Jagtman AM. Palatal wound healing: the effects of scarring on growth. En: Berkowitz S, editor. Cleft Lip and Palate. Berlin, Heidelberg, Germany: Springer; 2006. p.301-313.

15. Hochban W, Ganss C, Austermann KH. Long-term results after maxillary advancement in patients with clefts. Cleft Palate Craniofacial J. 1993;30(2):237-243. DOI:10.1597/1545-1569_1993_030_0237_ltrama_2.3.co_2.
16. Witzel MA, Munro IR. Velopharyngeal insufficiency after maxillary advancement. Cleft Palate J. 1977;14(2):176-180.

17. Ilizarov GA. The tension-stress effect on the genesis and growth of tissues. Part I. The influence of stability of fixation and soft-tissue preservation. Clin Orthop Relat Res. 1989;(238):249-281.

18. McCarthy JG, Schreiber J, Karp N, Thorne CH, Grayson $\mathrm{BH}$. Lengthening the human mandible by gradual distraction. Plast Reconstr Surg. 1992;89(1):1-8.

19. Cohen SR, Burstein FD, Stewart MB, Rathburn MA. Maxillary-midface distraction in children with cleft lip and palate: a preliminary report. Plast Reconstr Surg. 1997;99(5):1421-1428.

20. Polley JW, Figueroa AA. Management of severe maxillary deficiency in childhood and adolescence through distraction osteogenesis with an external, adjustable, rigid distraction device. J Craniofac Surg. 1997;8(3):181-185.

21. Figueroa AA, Polley JW. Orthodontics in cleft lip and palate management. En: Rodriguez ED, Losee JE, Neligan P, editores. Plastic Surgery and Pediatric Plastic Surgery. Philadelphia: Elsevier Saunders; 2013. p. 595-613.

22. KLS Martin Group. [Consultado el 20 de marzo 2019] Accesible en: http://www.klsmartinnorthamerica.com/ products/distraction-devices/lefort-i-and-ii/red-ii/

23. Doucet JC, Herlin C, Bigorre C, Bäumler G, Subsol G, Captier G. Mandibular effects of maxillary distraction osteogenesis in cleft lip and palate. Int J Oral Maxillof Surg. 2014;43(6):702-707. DOI: 10.1016/j. ijom.2014.01.017.

24. Meazzini Mc, Basile V, Mazzoleni F, Bozzetti A, Brusati R. Long-term follow-up of large maxillary advancements with distraction osteogenesis in growing and non-growing cleft lip and palate patients. J Plast Reconstr Aesthet Surg. 2015;68(1):79-86. DOI: 10.1016/j. bjps.2014.08.069.

25. Kim J, Uhm KI, Shin D, Lee J, Choi H. Maxillary distraction osteogenesis using a rigid external distractor: which clinical factors are related with relapse? J Craniofac Surg. 2015;26(4):1178-1181. DOI: $10.1097 /$ SCS.0000000000001568.

26. Jena AK, Rattan V, Singh S, Utreja A, Sombir S. LongTerm effect of maxillary distraction osteogenesis (DO) on nasal Index in adult with cleft lip and palate deformities. J Maxillofac Oral Surg. 2016;15(1):12-17. DOI: 10.1007/s12663-015-0794-1.

27. Sawada H, Ogawa T, Kataoka K, Baba Y, Moriyama K. Measurement of distraction force in cleft lip and palate patients during Le Fort I maxillary advancement with rigid external distraction. J Craniofac Surg. 2017;28(2):406412. DOI: $10.1097 /$ SCS.0000000000003327.

28. Painatt JM, Veeraraghavan R, Puthalath U, Peter S, Rao LP, Kuriakose M. Profile changes and stability following distraction osteogenesis with rigid external distraction in adult cleft lip and palate deformities. Contemp Clin Dent. 2017;8(2):236-243. DOI: 10.4103/ccd. ccd_1164_16.

29. Ahn JG, Figueroa AA, Braun S, Polley JW. Biomechanical considerations in distraction of the osteotomized 
dentomaxillary complex. Am J Orthod Dentofacial Orthop. 1999;116(3):264-270.

30. Gürsoy S, Hukki J, Hurmerinta K. Five-year follow-up of maxillary distraction osteogenesis on the dentofacial structures of children with cleft lip and palate. J Oral Maxillofac Surg. 2010;68(4):744-750. DOI: 10.1016/j. joms.2009.07.036.

31. Sahoo N, Desai AP, Jayan B, Gupta V. Evaluation of treatment outcome in cleft maxillary hypoplasia treated by rigid external distractor: a cephalometric study. J Craniofac Surg. 2014;25(1):143-148. DOI: 10.1097/ SCS.0000000000000374.

32. Sahoo NK, Jayan B, Roy ID, Desai A. Evaluation of the hard and soft tissue changes after maxillary advancement with rigid external distractor in unilateral cleft lip and palate induced maxillary hypoplasia. Med J Armed Forces India. 2015;71(2):355-361. DOI: 10.1016/j.mjafi.2013.12.016.

33. Dua G, Navin Kumar A, Roy ID, Roy SK. Maxillary distraction osteogenesis in cleft lip and palate cases with midface hypoplasia using rigid external distractor: an al- ternative technique. J Craniofac Surg. 2014;25(3):746751. DOI: 10.1097/SCS.0000000000000735.

34. Taha M, Elsheikh YM. Velopharyngeal changes after maxillary distraction in cleft patients using a rigid external distraction device: A retrospective study. Angle Orthod. 2016;86(6):962-968. DOI: 10.2319/011216-33.1.

35. Yeshwant KC, Seldin EB, Kikinis R, Kaban LB. A computer-assisted approach to planning multidimensional distraction osteogenesis. Atlas Oral Maxillofac Surg Clin North Am. 2005;13(1):1-12. DOI: 10.1016/j. cxom.2004.10.001.

36. Chang CS, Swanson J, Yu J, Taylor JA. Early, computer-aided design/computer-aided modeling planned, Le Fort I advancement with internal distractors to treat severe maxillary hypoplasia in cleft lip and palate. J Craniofac Surg. 2017;28(4):931-934. DOI: 10.1097/ SCS.0000000000003567.

37. Martínez A, Menéndez M, Martínez I, Fernández J, Gálvez P, Monsalve F. Avance maxilar en pacientes fisurados labio palatinos con distractor intraoral. Rev Esp Cirug Oral y Maxilofac. 2015;37(3):123-131. DOI: 10.1016/j. maxilo.2014.01.001. 